\author{
Advanced Studies in Biology, Vol. 6, 2014, no. 2, 47 - 56 \\ HIKARI Ltd, www.m-hikari.com \\ http://dx.doi.org/10.12988/asb.2014.4312
}

\title{
Fractional Order SEIRS Model
}

\author{
Muhammad Ozair*, Umer Saeed and Takasar Hussain \\ School of Natural Sciences \\ National University of Sciences and Technology \\ H-12 Campus, Islamabad 44000, Pakistan \\ * Corresponding author
}

\begin{abstract}
Copyright (C) 2014 Muhammad Ozair, Umer Saeed and Takasar Hussain. This is an open access article distributed under the Creative Commons Attribution License, which permits unrestricted use, distribution, and reproduction in any medium, provided the original work is properly cited.
\end{abstract}

\begin{abstract}
In this paper, we introduce a fractional order SEIRS model. The local asymptotic stability of equilibrium points is studied. We utilized the Adams-Bashforth predictor corrector method for solving the proposed model. Numerical simulations are presented to show the advantage of introducing a fractional model.
\end{abstract}

\section{Introduction}

Mathematical modeling of the spread of infectious diseases continues to be an area of active research. It has become an important tool in understanding the dynamics of diseases and in decision making processes regarding intervention programs for controlling these diseases in many countries. We replace the real world phenomena of the disease by an abstract model in order to employ the tools of mathematical analysis. This model typically takes a mathematical formulation that involves basic variables and relationships corresponding to the entities and the laws of nature or behavior being observed. It usually takes the form of non-linear ordinary differential equations. The subsequent solution is in the mathematical form and can be reinterpreted back in the original real world setting.

Mathematical modeling of complex biological processes is a major challenge for contemporary scientists. The complex systems are characterized by the variability of structures in them, multiscale behavior and nonlinearity in the mathematical description of the mutual relationship 
between parameters [1]. Therefore, the theory of integer-order differential equations is not sufficient to describe their dynamics.

In recent years, fractional differential equations become more popular because of its powerful potential applications. A large number of new differential equations models that involve fractional calculus are developed. There have found a number of works, especially in mechanics, biology, chemistry, electrical engineering $[2,3,4,5,6]$. In the literature, a number of methods have been developed for the numerical or analytical solutions for fractional differential equations. We listed some of these methods as follows: Adomian decomposition method [7], the collocation method [8], the fractional differential transform method [9, 10], homotopy analysis method [11, 12], homotopy perturbation method [13].

In biology, it has been deduced that the membranes of cells of biological organism have fractional-order electrical conductance and then are classified in groups of non-integer order models. Fractional derivatives embody essential features of the behavior of the pattern formation in bacterial colonies. Also, it has been shown that modeling the behavior of brainstem vestibule-oculumotor neurons by fractional ordinary differential equations has more advantages than classical integer-order modeling. Fractional derivatives are naturally related to systems with memory which exists in most biological systems [14]. For more details about using fractional calculus in modeling complex biomaterials, we refer the reader to [1] and the references therein. Adam Bashforth predictor corrector method has been proposed in $[15,16]$ for solving fractional differential equations. The method can be used for linear as well as nonlinear fractional differential equations. In the present work we use it for the numerical simulation of biological system which is fractional order. We introduce the Caputo derivative of order $\alpha$ in SEIRS model which was discussed in [17] and we discuss the oscillatory behavior of the endemic level of infected population. To the best of our knowledge, this work represents the first numerical solution for disease model of fractional order by applying the Adams-Bashforth-Moulton method. The rest of the paper is organized as follows. In section 2, some necessary definition and notations related to fractional calculus are presented. The formulation of the model and stability of equilibrium points is discussed in section 3. The general procedure for implementation of the predictor corrector method for the fractional model is discussed in section 4 and the numerical results are presented graphically in section 5. Finally, concluding remarks are given in section 6.

\section{Preliminaries}

First, we review some basic definitions of fractional differentiation and fractional integration [3].

\section{Riemann-Liouville fractional integral operator of order $\alpha$}

The operator $I_{x}^{\alpha}$, defined on $L_{1}[0, b]$ by

$I_{x}^{\alpha} y(x)=\frac{1}{\Gamma(\alpha)} \int_{0}^{x}(x-t)^{\alpha-1} y(t) d t$,

for $0 \leq x \leq b$, where $\alpha \in R^{+}$, is called Riemann-Liouville fractional integral of order $\alpha$. 
Riemann-Liouville and Caputo fractional derivative operator of order $\alpha$

The operator $D_{x}^{\alpha}$, defined by

$D_{x}^{\alpha} y(x)=\frac{1}{\Gamma(n-\alpha)}\left(\frac{d}{d x}\right)^{n} \int_{0}^{x}(x-t)^{n-\alpha-1} y(t) d t$,

for $0 \leq x \leq b$, where $\alpha \in R^{+}$and $n=\lceil\alpha\rceil$, is called Riemann-Liouville fractional integral of $\operatorname{order} \alpha$.The Caputo fractional derivative of a function $y \in L_{1}[0, b]$ is defined as

${ }^{c} D_{x}^{\alpha} y(x)=\frac{1}{\Gamma(n-\alpha)} \int_{0}^{x}(x-t)^{n-\alpha-1}\left(\frac{d}{d t}\right)^{n} y(t) d t$,

for $0 \leq x \leq b$, where $\alpha \in R^{+}$and $n=\lceil\alpha\rceil$.

\section{Mathematical Model}

The integer order model which is reported in [17] is given by

$$
\begin{aligned}
\frac{d S}{d t}=\pi+\theta R-\beta \frac{S I}{1+\omega N}-\mu S & \\
\frac{d E}{d t}=\beta \frac{S I}{1+\omega N} & -\sigma_{E} E-\mu E \\
\frac{d I}{d t} & =\sigma_{E} E-\sigma_{I} I-\mu I-\delta I \\
\frac{d R}{d t} & =\sigma_{I} I-\theta R-\mu R .
\end{aligned}
$$

This model has been discussed in the biologically feasible region $D=\left\{(S, E, I, R) \in R_{+}^{4}, S+E+I+R \leq \frac{\pi}{\mu}\right\}$ with initial conditions $S(0)>0, E(0)>0$, $I(0)>0, R(0)>0$. In this paper, we discuss the model consisting of fractional system of equations which is obtained by just replacing an integer order derivative by a fractional derivative of order $0<\alpha \leq 1$. Thus, the fractional order system is given by 


$$
\begin{aligned}
& D_{t}^{\alpha} S(t)=\pi+\theta R-\beta \frac{S I}{1+\omega N}-\mu S \\
& D_{t}^{\alpha} E(t)=\beta \frac{S I}{1+\omega N}-\sigma_{E} E-\mu E \\
& D_{t}^{\alpha} I(t)=\sigma_{E} E-\sigma_{I} I-\mu I-\delta I \\
& D_{t}^{\alpha} R(t)=\sigma_{I} I-\theta R-\mu R .
\end{aligned}
$$

\subsection{Asymptotic stability of Equilibrium Points}

To calculate the equilibrium points, we put the right hand side of system (2) equal to zero and obtain

$$
\begin{aligned}
\pi+\theta R^{*}-\beta \frac{S^{*} I^{*}}{1+\omega N^{*}}-\mu S^{*} & =0, \\
\beta \frac{S^{*} I^{*}}{1+\omega N^{*}}-\sigma_{E} E^{*}-\mu E^{*} & =0, \\
\sigma_{E} E^{*}-\sigma_{I} I^{*}-\mu I^{*}-\delta I^{*} & =0, \\
\sigma_{I} I^{*}-\theta R^{*}-\mu R^{*} & =0 .
\end{aligned}
$$

After simple calculation, we have

$$
\begin{aligned}
N^{*} & =\frac{\pi-\delta I^{*}}{\mu}, \\
R^{*} & =\frac{\sigma_{I} I^{*}}{\theta+\mu}, \\
E^{*} & =\frac{\left(\sigma_{I}+\mu+\delta\right)}{\sigma_{E}} I^{*}, \\
S^{*} & =\frac{\left(\sigma_{E}+\mu\right)\left(\sigma_{I}+\mu+\delta\right)\left(\mu+\omega\left(\pi-\delta I^{*}\right)\right)}{\mu \beta \sigma_{E}},
\end{aligned}
$$

and $I^{*}$ is the solution of the equation

$$
A I^{* 2}+B I^{*}+C=0
$$

where, 


$$
\begin{aligned}
& A=\left(-\theta \beta \mu \delta \omega \sigma_{I} \sigma_{E}\right)+\delta \omega(\theta+\mu)\left(\mu+\sigma_{E}\right)(\beta \mu-\mu \delta \omega)\left(\mu+\delta+\sigma_{I}\right), \\
& B=\left(-\beta \mu \sigma_{E}\left(\delta \omega(\pi \theta+\pi \mu)-\theta \sigma_{I}(\mu+\pi \omega)\right)\right) \\
& -(\theta+\mu)\left(\mu+\sigma_{E}\right)\left((\mu+\pi \omega)(\beta \mu-\mu \delta \omega)-\delta \omega\left(\mu^{2}+\pi \omega \mu\right)\right)\left(\mu+\delta+\sigma_{I}\right) \\
& C=\beta \mu \sigma_{E}(\pi \theta+\pi \mu)(\mu+\pi \omega)-(\theta+\mu)\left(\mu+\sigma_{E}\right)\left(\mu^{2}+\pi \omega \mu\right)(\mu+\pi \omega)\left(\mu+\delta+\sigma_{I}\right) .
\end{aligned}
$$

For positive equilibrium, it is clear from system (3) that $\pi-\delta I^{*}>0$, which means

$$
0<I^{*}<\frac{\pi}{\delta} .
$$

By using the condition (4) and the parameter values given in [17], the corresponding approximate equilibrium values of $S^{*}, E^{*}, I^{*}$ and $R^{*}$ are given in the following table.

Table 1: Equilibrium Values for different $\theta$ and $\delta$.

\begin{tabular}{cccccccccccc}
$\pi$ & $\theta$ & $\beta$ & $\omega$ & $\mu$ & $\sigma_{E}$ & $\sigma_{I}$ & $\delta$ & $S^{*}$ & $E^{*}$ & $I^{*}$ & $R^{*}$ \\
\hline 8 & 0 & 0.48 & 1 & 0.0027 & 0.5 & 0.2 & 0 & 1258 & 9 & 22 & 1672 \\
8 & 0.012 & 0.48 & 1 & 0.0027 & 0.5 & 0.2 & 0 & 1258 & 46 & 113 & 1544 \\
8 & 0 & 0.48 & 1 & 0.0027 & 0.5 & 0.2 & 0.0062 & 1275 & 9 & 21 & 1607 \\
8 & 0.012 & 0.48 & 1 & 0.0027 & 0.5 & 0.2 & 0.0062 & 1194 & 42 & 102 & 1389
\end{tabular}

\section{The Adams-Bashforth-Moulton Method}

We utilize Adams-Bashforth-Moulton predictor corrector method [15] for solving system of fractional order differential equations. The implementation of the adams-Bashforth-Moulton method according to the concerned fractional system is as follows:

$$
\begin{aligned}
& D^{\alpha} x(t)=g_{1}(t, x, y, z, u) \\
& D^{\alpha} y(t)=g_{2}(t, x, y, z, u) \\
& D^{\alpha} z(t)=g_{3}(t, x, y, z, u) \\
& D^{\alpha} u(t)=g_{4}(t, x, y, z, u),
\end{aligned}
$$


with $\quad x(0)=x_{0}, y(0)=y_{0}, z(0)=z_{0}, u(0)=u_{0}$, where $0<\alpha \leq 1$. Consider the uniform grid $t_{j}=j h, j=0,1, \ldots, N$ with some integer $N$ and $h=\frac{T}{N}$, where $T$ is the upper bound of the interval[ $[0, T]$. Implementation of the Adams-Bashforth Moulton method [15] on the proposed model are as follows:

Predictor values for (5) are

$$
\begin{aligned}
& x_{n+1}=x_{0}+\frac{h^{\alpha}}{\Gamma(\alpha+2)} g_{1}\left(t_{n+1}, x_{n+1}^{P}, y_{n+1}^{P}, z_{n+1}^{P}, u_{n+1}^{P}\right) \\
& +\frac{h^{\alpha}}{\Gamma(\alpha+2)} \sum_{j=0}^{n} a_{j},_{n+1} g_{1}\left(t_{j}, x_{j}, y_{j}, z_{j}, u_{j}\right) \\
& y_{n+1}=y_{0}+\frac{h^{\alpha}}{\Gamma(\alpha+2)} g_{2}\left(t_{n+1}, x_{n+1}^{P}, y_{n+1}^{P}, z_{n+1}^{P}, u_{n+1}^{P}\right) \\
& z_{n+1}=z_{0}+\frac{h^{\alpha}}{\Gamma(\alpha+2)} g_{3}\left(t_{n+1}, x_{n+1}^{P}, y_{n+1}^{P}, z_{n+1}^{P}, u_{n+1}^{P}\right) \\
& +\frac{h^{\alpha}}{\Gamma(\alpha+2)} \sum_{j=0}^{n} a_{j},_{n+1} g_{2}\left(t_{j}, x_{j}, y_{j}, z_{j}, u_{j}\right) \\
& \sum_{n+1}^{n}=u_{0}+\frac{h^{\alpha}}{\Gamma(\alpha+2)} g_{3}\left(t_{j}, x_{j}, y_{j}, z_{j}, u_{j}\right) \\
& \left.+\frac{h^{\alpha}}{\Gamma(\alpha+2)} \sum_{j=0}^{n} a_{j},_{n+1}, x_{n+1}^{P}, y_{n+1}^{P}, z_{n+1}^{P}, u_{n+1}^{P}\right) \\
& \left.t_{j}, x_{j}, y_{j}, z_{j}, u_{j}\right),
\end{aligned}
$$$$
\text { where } b_{j},_{n+1}=\frac{h^{\alpha}}{\alpha}\left((n-j+1)^{\alpha}-(n-j)^{\alpha}\right) \text {. }
$$

Corrector values are obtained by using Predictor values as 


$$
\begin{aligned}
x_{n+1}= & x_{0}+\frac{h^{\alpha}}{\Gamma(\alpha+2)} g_{1}\left(t_{n+1}, x_{n+1}^{P}, y_{n+1}^{P}, z_{n+1}^{P}, u_{n+1}^{P}\right) \\
& +\frac{h^{\alpha}}{\Gamma(\alpha+2)} \sum_{j=0}^{n} a_{j},_{n+1} g_{1}\left(t_{j}, x_{j}, y_{j}, z_{j}, u_{j}\right) \\
y_{n+1}= & y_{0}+\frac{h^{\alpha}}{\Gamma(\alpha+2)} g_{2}\left(t_{n+1}, x_{n+1}^{P}, y_{n+1}^{P}, z_{n+1}^{P}, u_{n+1}^{P}\right) \\
& +\frac{h^{\alpha}}{\Gamma(\alpha+2)} \sum_{j=0}^{n} a_{j},_{n+1} g_{2}\left(t_{j}, x_{j}, y_{j}, z_{j}, u_{j}\right) \\
z_{n+1}= & z_{0}+\frac{h^{\alpha}}{\Gamma(\alpha+2)} g_{3}\left(t_{n+1}, x_{n+1}^{P}, y_{n+1}^{P}, z_{n+1}^{P}, u_{n+1}^{P}\right) \\
& +\frac{h^{\alpha}}{\Gamma(\alpha+2)} \sum_{j=0}^{n} a_{j},_{n+1} g_{3}\left(t_{j}, x_{j}, y_{j}, z_{j}, u_{j}\right) \\
u_{n+1}= & u_{0}+\frac{h^{\alpha}}{\Gamma(\alpha+2)} g_{4}\left(t_{n+1}, x_{n+1}^{P}, y_{n+1}^{P}, z_{n+1}^{P}, u_{n+1}^{P}\right) \\
& +\frac{h^{\alpha}}{\Gamma(\alpha+2)} \sum_{j=0}^{n} a_{j},_{n+1} g_{4}\left(t_{j}, x_{j}, y_{j}, z_{j}, u_{j}\right)
\end{aligned}
$$

where

$$
a_{j, n+1}=\left\{\begin{array}{c}
n^{\alpha+1}-(n-\alpha)(n+1)^{\alpha}, j=0 \\
(n-j+2)^{\alpha+1}+(n-j)^{\alpha+1}-2(n-j+1)^{\alpha+1}, 1 \leq j \leq n
\end{array}\right.
$$

According to the mathematical analysis of this method in [16] we have order of accuracy " $p$ " where $p=\min (2,1+\alpha)$.

\section{Numerical results}

In this section, we shall discuss the oscillatory behavior of the endemic level of infected population of model (2), on the basis of the numerical results which are obtained by using the parameter 
values given in Table 1. It is clear from figure 1 that when the recovered individuals lose their infection-acquired immunity and in the absence of disease related death, there are damped oscillations of the infected populations for $\alpha=1$. The endemic equilibrium level is eventually attained and this converges to a steady state that is asymptotically stable. The oscillations decrease for fractional values of $\alpha$ and we obtain the endemic level at the early stage as compared to the integer order. Similar behavior has been observed when we suppose permanent immunity and disease related death rate of infected individuals. This behavior of infected population is shown in figure 2 .

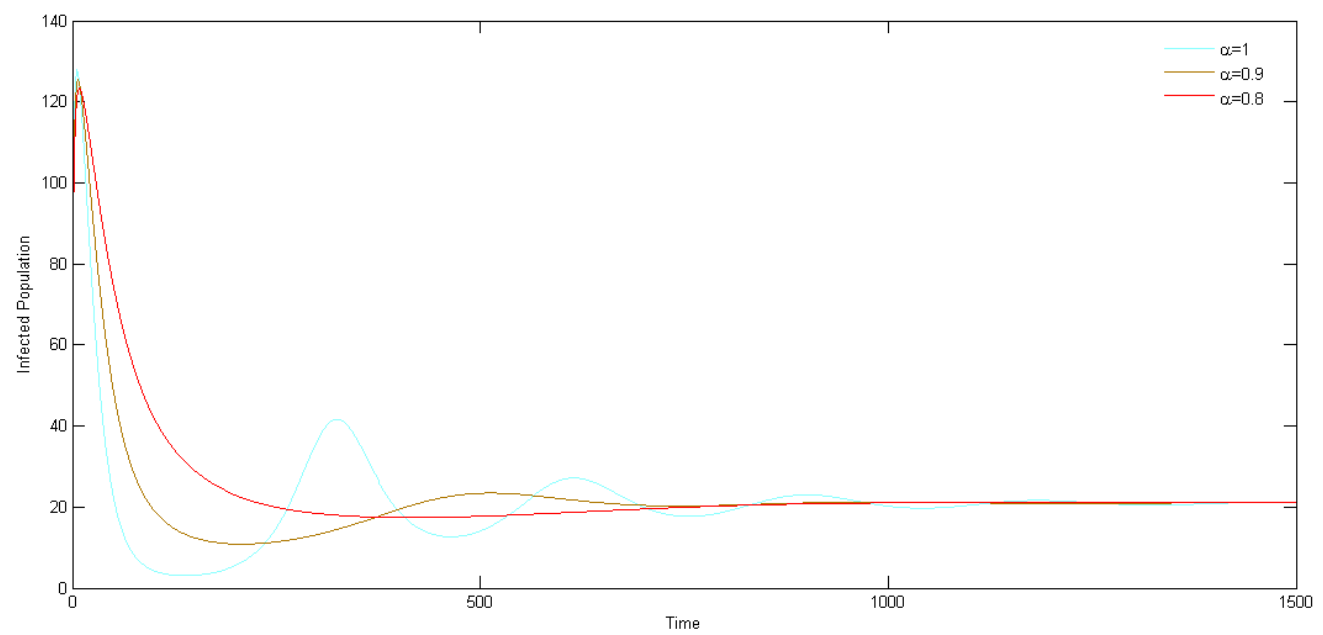

Figure1. Oscillatory behavior of infected population when $\theta=0.012$ and $\delta=0$.

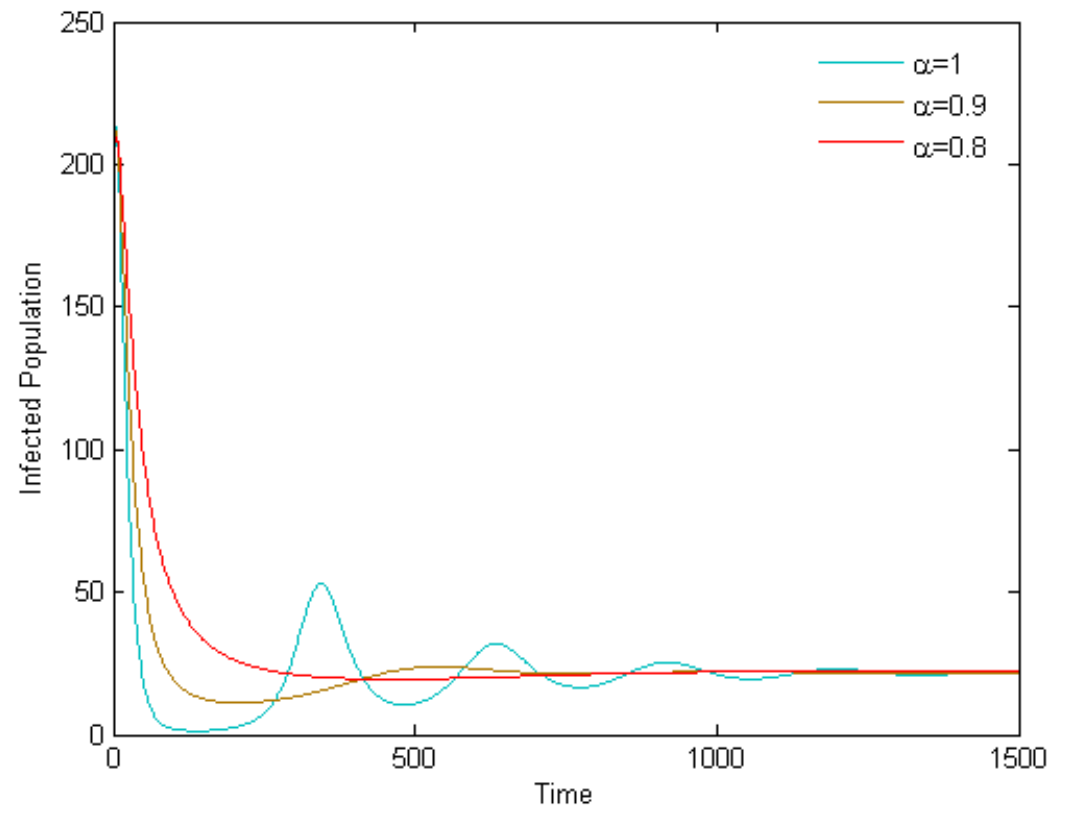

Figure2. Oscillatory behavior of infected population when $\theta=0$ and $\delta=0.0062$. 


\section{Discussion}

We reconsidered SEIR model, which was discussed in [17], by introducing the Caputo derivative of order $\alpha$ in place of ordinary derivative. We derive the relations of equilibrium values in terms of infected populations and the equilibrium points are obtained by using parameter values. Numerical simulations are carried out by using Adams-Bashforth-Moulton method and it is shown that fractional model provides better stability results and the endemic level is obtained at the early stage than the ordinary differential equations model. Thus the present success of the proposed fractional order model verifies that it is a useful tool for these kinds of models.

\section{References}

1) J. S. Leszczynski, An Introduction to Fractional Mechanics, Czestochowa University of Technology, 2011.

2) K. B. Oldham \& J. Spanier, The fractional calculus, Academic Press, New York (1974).

3) I. Podlubny, Fractional differential equations, Academic Press, San Diego (1999).

4) M. F. Shlesinger, G. M. Zaslavsky, \& J. Klafter, Strange kinetics, Nature, Vol. 363 No. 6,pp. 31-37 (1993).

5) S. G. Samko, A. A. Kilbas \& O. I. Marichev, Fractional Integrals and Derivatives: Theory and Applications, Gordon and Breach, London (1993).

6) Y. Ding and H. Ye, “A fractional-order differential equation model of HIV infection of CD4 巨 T-Cells”, Mathematical and Computer Modelling, Volume 50, Issues 3-4, August 2009, pages 386-392.

7) S. Momani and R. Qaralleh, "An efficient method for solving systems of fractional integro-differential equations," Computers \& Mathematics with Applications, vol. 52, no. 3-4, pp. 459-470, 2006.

8) E. A. Rawashdeh, "Numerical solution of fractional integro-differential equations by collocation method," Applied Mathematics and Computation, vol. 176, no. 1, pp. 1-6, 2006.

9) A. Secer, M. A. Akinlar, and A. Cevikel, "Efficient solutions of systems of fractional PDEs by the differential transform method," Advances in Difference Equations, vol. 2012, article 188, 2012.

10) A. Alawneh, "Application of the Multistep Generalized Differential Transform Method to Solve a Time-Fractional Enzyme Kinetics,' Discrete Dynamics in Nature and Society, Volume 2013 (2013), Article ID 592938, 7 pages.

11) M. Kurulay, A. Secer, and M. A. Akinlar, "A new approximate analytical solution of Kuramoto-Sivashinsky equation using homotopy analysis method," Applied Mathematics \& Information Sciences, vol. 7, no. 1, pp. 267-271, 2013. 
12) S. Z. Rida, A. A. M. Arafa and M. Khalil, "Solutions of Fractional model of human T-cell

lymphotropic virus I (HTLV-I) Infection of CD4 T-Cells using HAM,' International Journal of Basic and Applied Sciences, 1(1) (2012) 1-14.

13) Q.Wang, "Homotopy perturbation method for fractional KdVBurgers equation," Chaos, Solitons \& Fractals, vol. 35, no. 5, pp. 843-850, 2008.

14) A. A. M. Arafa, S. Z. Rida, M. Khalil, " Fractional Order Model of Human T-cell

Lymphotropic Virus I (HTLV-I) Infection of CD4 ${ }^{\complement}$ T-Cells,' Advanced Studies in Biology, Vol. 3, 2011, no. 7, 347 - 353.

15) K. Diethelm, N. J. Ford, A. D. Fareed, A predictor-corrector approach for the numerical solution of fractional differential equations, Nonlinear Dynamics 29:3-22, 2002.

16) K. Diethelm, N. J.Ford, A. D. Fareed, Detailed error analysis for a fractional Adams method, Numerical Algorithms 36: 31-52, 2004.

17) Shulin Sun, Cuihua Guo, Chengmin Li, Global Analysis of an SEIRS Model with Saturating Contact Rate, Applied Mathematical Sciences, Vol.6, 2012, no.80, 3991-4003.

\section{Received: March 17, 2014}

